Pacific Journal of Mathematics

HOMEOMORPHISMS OF MANIFOLDS WITH
ZERO-DIMENSIONAL SETS OF NONWANDERING POINT 


\title{
HOMEOMORPHISMS OF MANIFOLDS WITH ZERO-DIMENSIONAL SETS OF NONWANDERING POINTS
}

\author{
L. S. Husch and Ping-Fun Lam
}

Let $h$ be a self-homeomorphism of a compact $n$-dimensional manifold $M$, which is not homeomorphic to an odd dimensional sphere, such that the set $N$ of irregular points of $h$ is closed in $M$ and the set of nonwandering points of $h$ is zero-dimensional. One of the main results of this paper is that $N$ is either connected or consists of the two fixed points of $h$. In the latter case, $N$ is homeomorphic to the $n$-sphere. In the former case when $n=2$, it is shown that each component of $M-N$ is an open 2-cell. If $M$ is open and $N$ is compact, then it is shown that $M$ is homeomorphic to Euclidean $n$-space and $N$ consists of a single fixed point of $h$.

Let $h$ be a self-homeomorphism of a metric space $X$ with metric $d$. $\quad h$ is regular at $x \in X$ if, for every $\varepsilon>0$, there exists $\delta>0$ such that whenever $y \in X$ and $d(x, y)<\delta$, then $d\left(h^{n}(x), h^{n}(y)\right)<\varepsilon$ for all integers $n . \quad h$ is irregular at $x \in X$ if $h$ is not regular at $x$. Let $E(X, h)$ and $N(X, h)$ denote the set of regular and irregular points, respectively of $X$. This definition was originally given by Kerékjártó [10]. If $X$ is locally compact and connected, it was shown by Homma and Kinoshita [7] that if $N(X, h)$ is a finite set which does not separate $X$, then $N(X, h)$ consists of the fixed points of $h$ and contains at most two points. Kaul [9] obtained a similar result with the assumption that $X$ be locally connected and $N(X, h)$ be compact zero-dimensional and not separating $X$. In [12], [14] Lam obtained analogous results when $N(X, h)$ need not be zero-dimensional and $E(X, h)$ has a property called indivisibility. The property of indivisibility is generally weaker than the property that $E(X, h)$ be connected. As a result of [12], Kaul's theorem can be shown to be valid without assuming that $X$ be locally connected (cf. Theorem 2.11 in the following); hence, Kaul's theorem is a generalization of the theorem of Homma and Kinoshita. If $X$ is an open connected manifold, $N(X, h)$ is a compact set not separating $X$ and if $h$ is positively regular on all of $X$, then it was shown by Duvall and Husch [4] that $N(X, h)$ is a strong deformation retract of $X$ and, hence, must be connected.

A point $x \in X$ is nonwandering or regionally recurrent under $h$, if for every neighborhood $U$ of $x$ there exists a subsequence $\left\{m_{i}\right\}_{i=1}^{\infty}$ of the positive integers such that $h^{m}(U) \cap U \neq \varnothing$; let $R(X, h)$ be the set of all nonwandering points of $h$ in $X$. One of the steps in Homma 
and Kinoshita's and Kaul's theorems is equivalent to showing that $R(X, h) \subseteq N(X, h)$ and hence the zero-dimensionality of $N(X, h)$ implies that of $R(X, h)$. In this paper, we assume the zero-dimensionality of $R(X, h)$ and obtain results which may be considered as generalizations of [7] and [9] on phase spaces which are manifolds. Actually, as our proofs will show, the hypothesis that $R(X, h)$ is zero-dimensional can be replaced by the weaker hypothesis that the set of all $\omega$-limiting points and $\alpha$-limiting points is zero-dimensional. We give examples in $\S 6$ to demonstrate the extent of our generalizations.

THEOREM A. Let $h$ be a self-homeomorphism of a closed n-dimensional manifold $M$ such that the set $N(M, h)$ of irregular points of $h$ is a closed nonempty subset of $M$. If the set $R(M, h)$ of nonwandering points is zero-dimensional, then $R(M, h) \subseteq N(M, h)$ and

(a) $N(M, h)$ is a continuum provided $M$ is not homeomorphic to the n-sphere;

(b) $N(M, h)$ is either a continuum or consists of the two fixed points of $h$ when either $M$ is homeomorphic to an even dimensional sphere or $M$ is homeomorphic to an odd dimensional sphere and $h$ is periodic at some $x \in M$.

Theorem B. If, in addition to the hypothesis of Theorem A, $n=2$ and $N(M, h)$ is a continuum, then each component of $M$ $N(M, h)$ is an open 2-cell.

Theorem C. If, in addition to the hypothesis of Theorem A, M is orientable, then, using homology and cohomology with field coefficients, for all $q<n, \bar{H}^{q}(N(M, h))$ is isomorphic to the direct sum of

$$
\tilde{H}_{n-q-1}(E(M, h)) \text { and } H_{n-q}(M) \text {. }
$$

THEOREM D. If, in the hypothesis of Theorem A, the assumptions that $M$ is a closed manifold and $N(M, h)$ is closed are replaced by the assumption that $M$ is an open manifold and $N(M, h)$ is compact, then $R(M, h)=N(M, h)=$ the fixed point of $h$ and $M$ is homeomorphic to Euclidean n-space.

In this paper a manifold is a connected separable locally Euclidean metric space. Manifolds are therefore assumed to have no boundary unless the otherwise is stated.

2. Preliminary results on metric spaces. Throughout this section we let $X$ be a metric space with metric $d$ and let $h$ be a selfhomeomorphism of $X$. Whereas, in general, the regularity of $h$ at 
$x \in X$ depends upon the metric $d$, if $X$ is compact, the regularity of $h$ at $x \in X$ is independent of the metric.

Proposition 2.1. For all integers $k \neq 0$,

$$
E\left(X, h^{k}\right)=E(X, h), N\left(X, h^{k}\right)=N(X, h)
$$

and

$$
R\left(X, h^{k}\right) \subseteq R(X, h)
$$

provided $X$ is compact.

Proposition 2.2. The following properties hold.

(1) If $K$ is a minimal set in $X$, then either $K \subseteq E(X, h)$ or $K \cong N(X, h)$.

(2) If $X$ is locally compact, then the set of minimal sets contained in $E(X, h)$ is relatively closed in $E(X, h)$.

This proposition, like some of the following statements, is proved under more general setting in [13]. For a proof of (1) and (2) see Remark 1.6 and Corollary 3.6, respectively, of [13].

Proposition 2.3. If $X$ is locally compact separable and $N(X, h)$ is compact, then the set $E(X, h)$ is unchanged when the original metric is replaced by a metric induced from the one-point compactification of $X$.

Proof. By the definition of irregular points and the compactness of $N(X, h)$ we observe that $N(X, h)$ is at least as large after the change of metric. That $N(X, h)$ is at most as large after the change of metric is proved in Proposition 2.11 of [13] or in [18].

A point $x \in X$ is almost periodic if, for every $\varepsilon>0$, there exists a subsequence of integers, $\cdots<n_{-1}<n_{0}<n_{1}<\cdots$ and a constant $c$ such that $\left|n_{i}-n_{i+1}\right|<c$ for all integers $i$ and $d\left(x, h^{m_{i}}(x)\right)<\varepsilon$ for all $i$. If $x \in X, y \in \omega(x)(y \in \alpha(x))$ if there exists a subsequence $\left\{n_{i}\right\}_{i=1}^{\infty}$ of the positive (negative) integers such that $\lim _{i \rightarrow+\infty} h^{n i}(x)=y$. We now state two propositions which follow from Lemmas 2 and 4 of [7].

Proposition 2.4. If $x \in E(X, h)$, then $x$ is nonwandering if and only if $E(X, h) \cap[\alpha(x) \cup \omega(x)] \neq \varnothing$.

Proposition 2.5. If $X$ is locally compact and if $p$ is an interior point of $E(X, h)$, then, $p$ is nonwandering if and only if $p$ is almost periodic. 
Proposition 2.6. If $X$ is locally compact and $x \in X$, then $x$ is almost periodic if and only if the orbit closure of $x$

$$
\text { (-i.e., }\left\{\mathrm{Cl}\left(h^{n}(x) \mid-\infty<n<+\infty\right\}\right)
$$

is a compact minimal set.

The proof of Proposition 2.6 can be found in [6; pp. 31-32].

Proposition 2.7. Let $X$ be locally compact separable and $N(X, h)$ be compact. If $Y \leqq E(X, h)$ is connected, then either $Y$ has no nonwandering points or all points in $Y$ are nonwandering.

Proof. Let $A$ be the set of nonwandering points of $Y$. By Proposition 2.5, $A$ is the set of almost periodic points of $Y$ and hence, by Proposition 2.6, each point of $A$ lies in a compact minimal set which lies in $E(X, h)$ by Proposition 2.2 (1). Let $X^{*}=X \cup\{\infty\}$ be the one-point compactification of $X$ and extend $h$ to a self-homeomorphism of $X^{*}$ by letting $h(\infty)=\infty$. Let $d^{*}$ be a compatible metric of $X^{*}$. By Proposition 2.3, $E\left(X^{*}, h\right) \supseteqq E(X, h)$. Now if $p \in Y$ is a limit point of $A$, then by Proposition 2.2 (2), applied to the phase space $X^{*}$, we have that the orbit closure of $p$ in $X^{*}$ is a minimal set. It follows from Proposition 2.6 that $p$ is almost periodic. Hence the set $A$ is relatively closed in $Y$. If $U$ is a sufficiently small neighborhood of $p \in A$, then by Propositions 2.5 and 2.6 the regularity of $h$ at $p$ and the openness of $E(X, h)$, the orbit closure of every $q \in U$ is a compact set in $E(X, h)$. It follows from Proposition 2.4 that $U \cap Y \subseteq A$. Hence $A$ is relatively open in $Y$. Since the set $Y$ is assumed to be connected we must have either $A=Y$ or $A=\Phi$. The assertion in Proposition 2.7 then follows.

Lemma 2.8. Suppose that $X$ is locally compact separable and that $N(X, h)$ is compact. Let $Y$ be a connected subset of $E(X, h)$. Then for every sequence of distinct integers $\left\{n_{i}\right\}_{i=1}^{\infty}$ the following property holds. If there exists $y_{0} \in Y$ such that the sequence $\left\{h^{n_{i}}\left(y_{0}\right)\right\}_{i=1}^{\infty}$ has a limit point in $E(X, h)$, then $\left\{h^{n i}(y)\right\}_{i=1}^{\infty}$ has a limit point in $E(X, h)$ for all $y \in Y$.

Proof. The lemma is obtained in [13] under the general setting of indivisibility. We give a different proof here, which is based on the propositions just obtained. Let $y \in Y$ and $\left\{n_{i}\right\}_{i=1}^{\infty}$ the given sequence of distinct integers. It is clear that the sequence $\left\{h^{n_{i}}(y)\right\}_{i=1}^{\infty}$ has a limit point in $E(X, h)$ if and only if $E(X, h) \cap[\alpha(y) \cup \omega(y)] \neq \varnothing$. According to Proposition 2.4 this is so if and only if $y$ is nonwandering. Lemma 2.8 then follows from Proposition 2.7. 
We remark that the assumption that $X$ be separable in Proposition 2.7 and Lemma 2.8 can be removed by appealing to techniques of uniform spaces.

LEMMA 2.9. Suppose that $X$ is locally compact and connected and $N(X, h)$ is compact. If $A(X, h)$, the set of almost periodic points, is totally disconnected, then $\alpha(x) \cup \omega(x) \subseteq N(X, h)$ for all $x \in E(X, h)$.

Proof. Suppose that there exist $x_{0}, x_{1} \in E(X, h)$ such than $x_{1} \in$ $\alpha\left(x_{0}\right) \cup \omega\left(x_{0}\right)$ and we seek a contradiction. Without loss of generality we may assume that $x_{1} \in \omega\left(x_{0}\right) \cap E(X, h)$. It follows from Proposition 2.4 that $x_{0}$ is nonwandering. By Proposition 2.5 and the closedness of $N(X, h)$ we have that $x_{0}$ is almost periodic. By Proposition 2.6 the orbit closure $K$ of $x_{0}$ is a compact minimal set. By Proposition 2.2 (1) we conclude that $K \subseteq E(X, h)$. It follows that both $\alpha\left(x_{0}\right)$ and $\omega\left(x_{0}\right)$ are compact and $\alpha\left(x_{0}\right) \cup \omega\left(x_{0}\right) \subseteq E(X, h)$. Let $Y$ be the component of $E(X, h)$ which contains $x_{0}$. If we apply Lemma 2.8 by setting $y_{0}$ to be $x_{0}$ and by taking arbitrary sequences of distinct integers $\left\{n_{i}\right\}_{i=1}^{\infty}$, we find that $\alpha(x) \cup \omega(x) \subseteq E(X, h)$ for all $x \in Y$. (Note that a locally compact connected metric space is separable.) It follows from Propositions 2.4 and 2.5 that $Y \subseteq A(X, h)$. Since $A(X, h)$ is assumed to be totally disconnected, we have that the component $Y$ of $E(X, h)$ is a singleton. This is impossible due to the fact that $X$ is connected and $N(X, h)$ is closed (a compact component of the locally compact space $E(X, h)$ has arbitrarily near relatively open and closed neighborhoods). We then have a contradiction.

The proof of Lemma 2.9 is completed.

Lemma 2.10. If $X$ and $N(X, h)$ are compact, $X$ is connected and $R(X, h)$ is zero-dimensional, then a continuum $K$ in $E(Y, h)$ has the following property. For every subsequence of integers $\cdots n_{-1}<n_{0}<$ $n_{1}<\cdots$ there exists points $p, q \in N(X, h)$, not necessarily distinct, and a subsequence $\left\{m_{k}=n_{i_{k}}\right\}_{k=-\infty}^{+\infty}$ of $\left\{n_{i}\right\}_{i=-\infty}^{+\infty}$ such that

$$
\lim _{k \rightarrow+\infty} h^{m_{k}}(x)=p, \lim _{k \rightarrow-\infty} h^{m_{k}}(x)=q \text { for all } x \in K .
$$

Moreover, the convergence of the two sequences are uniform on $K$.

Proof. Let the sequence $\left\{n_{i}\right\}_{i=1}^{\infty}$ be obtained from the sequence $\left\{n_{i}\right\}_{i=-\infty}^{+\infty}$. Let $\mathscr{C}$ denote the space of all continuous maps from $K$ to $X$ with the sup metric. If we apply the Arzela-Ascoli Theorem $\left[3\right.$, p. 267] to the sequence of maps $\left\{h^{n i}\right\}_{i=1}^{\infty}$ with their domains restricted to $K$, we obtain a sequence in $\mathscr{C}$ which converges. Since $\mathscr{C}$ is complete, we have a subsequence $\left\{m_{k}=n_{i_{k}}\right\}_{k=1}^{\infty}$ of $\left\{n_{i}\right\}_{i=1}^{\infty}$, with domains 
again restricted to $K$, which converges to some $f \in \mathscr{C}$. Let $x_{1}$ be an arbitrary point of $K$ and let $p=\lim _{k \rightarrow \infty} h^{m_{k}}\left(x_{1}\right)$. Then $p \in \omega\left(x_{1}\right)$, and from Lemm $2.9 p \in N(X, h)$. We claim that the sequence $\left\{h^{m_{k}}(x)\right\}_{k=1}^{\infty}$ converges uniformly to $p$ for $x \in K$.

For if the claim fails then, because of the connectedness of $K$, for a sufficiently small neighborhood $U$ of $p$ we have that $\partial U \cap h^{m_{k}}(K) \neq$ $\varnothing$ for infinitely many terms in $\left\{m_{k}\right\}_{k=1}^{\infty}$. By the hypothesis that $R(X, h)$ is zero-dimensional and by the hereditary normal property of the metric space $X$ one can find arbitrarily small neighborhoods $V$ of $p$ such that $\partial V \cap R(X, h)=\varnothing$. We may restrict neighborhoods $U$ above to satisfy this condition. There then exist sequences $\left\{y_{j}\right\} \subseteq K,\left\{z_{j}\right\} \leqq \partial U$, points $y \in K, z \in \partial U$ and a subsequence $\left\{\alpha_{j}=m_{k_{j}}\right\}_{j=1}^{\infty}$ of $\left\{m_{k}\right\}_{k=1}^{\infty}$ such that

$$
z_{j}=h^{\alpha}\left(y_{j}\right), y=\lim _{j \rightarrow \infty} y_{j}, z=\lim _{j \rightarrow \infty} z_{j} .
$$

Since $y \in E(X, h)$, it follows that

$$
\lim _{j \rightarrow \infty} h^{\alpha} j(y)=\lim _{j \rightarrow \infty} h^{\alpha}\left(y_{j}\right)=\lim _{j \rightarrow \infty} z_{j}=z .
$$

Hence $z \in R(X, h) \cap \partial U$. This contradicts that $\partial U \cap R(X, h)=\varnothing$. Our claim is thus valid. If we apply a similar argument to the sequence $\left\{n_{-i}\right\}_{i=1}^{\infty}$, we obtain the point $q$ and the corresponding properties asserted in the lemma.

The proof of Lemma 2.10 is completed.

In order to prove the next lemma we give a generalization of the theorems of [7], [9].

TheOREM 2.11. If $X$ is a compact and connected space, $N(X, h)$ is zero-dimensional (nonempty) and $E(X, h)$ contains an open, dense connected and invariant subset $D$, then $N(X, h)$ equals the union of one or two fixed points of $h$. Moreover, there exist $p, q \in N(X, h)$, not necessarily distinct, such that

$$
\lim _{n \rightarrow+\infty} h^{n}(x)=p, \lim _{n \rightarrow-\infty} h^{n}(x)=q \text { for all } x \in E(X, h) .
$$

Proof. The proof is a direct application of Theorems 2 and 3 of Lam [12]. The theorems are applied to the discrete flow $(X, Z)$ generated by $h(Z$ : additive group of integers). Since the set $D$ is now open, it is locally compact. According to Theorem 3 (II) of [12] the set $E(X, h)$ is indivisible by $Z$. By Theorem 2 of [12], applied to the abelian group $Z$, the set $N(X, h)$ is a union of one or two fixed points. Moreover, there exists a sequence $\left\{t_{i}\right\}$ in $Z$ and points $p, q \in N(X, h)$, not necessarily distinct, such that

$$
\lim _{i \rightarrow \infty} h^{t_{i}}(x)=p, \lim _{i \rightarrow \infty} h^{-t_{i}}(x)=q \text { for all } x \in E(X, h) .
$$


Since $x \notin N(X, h), \lim _{i \rightarrow \infty}\left|t_{i}\right|=+\infty$. Without loss of generality we let $\lim _{i \rightarrow \infty} t_{i}=+\infty$. We claim that $\lim _{n \rightarrow \infty} h^{n}(x)=p$. For otherwise we would obtain some $z \in \omega(x) \cap E(X, h)$ near $p$, using the fact that $p$ is a fixed point. By (2.4), (2.5), (2.6), and (2.2(1)) the orbit closure of $x$ then is in $E(X, h)$, which would contradict $p \in \omega(x)$. Hence $\lim _{n \rightarrow \infty} h^{n}(x)=p$. Likewise we have

$$
\lim _{n \rightarrow-\infty} h^{n}(x)=q \text { for all } x \in E(X, h) .
$$

Theorem 2.11 follows.

Let $(S, Z)$ be a discrete flow on a metric space $S$. If there exist two nonempty, closed and invariant subsets $E$ and $F$ such that $S=$ $E \cup F$ and $E \cap F=\varnothing$, then $S$ is said to be dynamically disconnected.

Lemma 2.12. Suppose that $X$ is compact connected and that $E(X, h)$ is connected and has at least one point which is not almost periodic. If $N(X, h)$ is compact and dynamically disconnected, then the following properties hold:

(1) $N(X, h)$ consists of two components $H$ and $K$.

(2) Either we have lim sup $\sup _{n \rightarrow+\infty}\left\{h^{n}(x)\right\} \subseteq H$ and $\lim \sup _{n \rightarrow-\infty}\left\{h^{n}(x)\right\} \subseteq$ $K$ for all $x \in E(X, h)$ or we have lim $\sup _{n \rightarrow+\infty}\left\{h^{n}(x)\right\} \subseteq K$ and

$$
\lim _{n \rightarrow-\infty} \sup \left\{h^{n}(x)\right\} \subseteq H \text { for all } x \in E(X, h) .
$$

Proof. We form the quotient space $X_{*}$ of $X$ by identifying components of $N(X, h)$ as points. Since the decomposition is upper semicontinuous, the compact space $X_{*}$ is metrizable. A homeomorphism $f: X_{*} \rightarrow X_{*}$ (onto) is induced from $h$ by requiring that $f \circ \pi=\pi \circ h$, where $\pi$ is the canonical projection of $X$ onto $X_{*}$. We give $X_{*}$ a metric and define sets $E\left(X_{*}, f\right)$ and $N\left(X_{*}, f\right)$. From the uniform continuity of $\pi$ and the openness of $\pi \mid E(X, h)$ we have $\pi(E(X, h)) \subseteq$ $E\left(X_{*}, f\right)$. We claim $N\left(X_{*}, f\right) \neq \varnothing$. For, by our hypothesis and Propositions 2.5 and 2.7 , the set $E(X, h)$ contains only wandering points. By Proposition 2.4, we have $\alpha(x) \cup \omega(x) \subseteq N(X, h)$ for all $x \in E(X, h)$. It follows that $\alpha(z) \cup \omega(z) \subseteq \pi(N(X, h))$ for all $z \in \pi(E(X, h))$. Hence $\pi(N(X, h)) \cap N\left(X_{*}, f\right) \neq \varnothing$. Now $\pi(E(X, h))$ is open, connected, dense in $E\left(X_{*}, f\right)$ and invariant. By Theorem 2.11, we can conclude that $N\left(X_{*}, f\right)$ equals the union of one or two fixed points and obtain points $p, q \in N\left(X_{*}, f\right)$, not necessarily distinct, such that

$$
\lim _{n \rightarrow+\infty} f^{n}(x)=p, \lim _{n \rightarrow-\infty} f^{n}(x)=q \text { for all } x \in E\left(X_{*}, f\right) .
$$

Let $H$ and $K$ now be two disjoint nonempty compact and invariant subsets of $X$ whose union is $N(X, h)$. Then $\pi(H)$ and $\pi(K)$ constitute two disjoint compact invariant subsets in $X_{*}$. According to property 
(a) we must have $\pi(H)=p$ and $\pi(K)=q$ or $\pi(H)=q$ and $\pi(K)=p$. Hence $H$ and $K$ are connected and $\pi(N(X, h))=N\left(X_{*}, f\right)$. Lemma 2.12 then follows.

3. Structure of the set of irregular points. If $U$ is an open connected manifold, then $U$ has one end if, for each compact subset $C$ of $U$, there exists a compact set $D$ such that $C \cong D \subseteq U$ and $U-D$ is connected.

Proposition 3.1. If $F$ is a metric continuum and $G$ is an open subset of $F$ such that each component of $G$ is a manifold with one end, then $F-G$ is connected.

Proof. $G$ has a countable number of components, say $G_{i}, i=1$, $2, \cdots$. Since each $G_{i}$ has one end and $G_{i}$ is separable, there exists a countable sequence of compacta, $\left\{C_{i, j}\right\}_{j=1}^{\infty}$, such that $C_{i, j} \leqq \operatorname{int} C_{i, j+1} \leqq$ $G_{i}, G_{i}-C_{i, j}$ is connected, and $G_{i}=\bigcup_{j=1}^{\infty} C_{i, j}$ for all $i$ and $j$. Let $\left\{D_{k}\right\}_{k=1}^{\infty}=\left\{C_{i, j}\right\}_{i, j=1}^{\infty}$ be a reindexing. By inductive argument the sets $F-\bigcup_{k=1}^{n} D_{k}$ are connected for all $n=1,2, \cdots, F-G$ can then be expressed as the intersection of a nested sequence of continua, $\{\mathrm{Cl}(F-$ $\left.\left.\bigcup_{k=1}^{n} D_{k}\right)\right\}_{n=1}^{\infty}$, and hence is connected.

Proposition 3.2. If $M$ is a closed n-manifold and if $M$ can be expressed as the union of two open n-cells, then $M$ is homeomorphic to the n-sphere.

Proof. This follows from [2]; see the Appendix of [11].

Proposition 3.3. Let $h$ be a self-homeomorphism of a closed $n$ manifold $M$ such that $N(M, h)$ is a closed nonempty set and $R(M, h)$ is zero-dimensional. If $M$ is not homeomorphic to the n-sphere, then each component of $E(M, h)$ has one end.

Proof. Let $D$ be a component of $E(M, h)$ and suppose that $D$ does not have one end; - i.e., there exists a compact set $C \cong D$ such that for each compact set $K, C \subseteq K \subseteq D, D-K$ is not connected. By Lemma 2.10, there exists a subsequence $\left\{m_{k}\right\}_{k=-\infty}^{+\infty}$ of the integers and points $p$ and $q$ such that $\lim _{k \rightarrow \infty} h^{m_{k}}(C)=p$ and $\lim _{k \rightarrow-\infty} h^{m_{k}}(C)=q$. Hence $h^{m_{k}}(C), k$ sufficiently large, lies in an open $n$-cell $U$ in $M$. By replacing $D$ with $h^{m_{k}}(D)$ we may assume that $C \subset U$. It follows that some neighborhood of $C$ in $U \cap E(M, h)$ can be triangulated as a piecewise linear manifold and hence there exists a compact $n$-manifold $W$ with boundary such that $C \leqq W \sqsubseteq U \cap D$. By the choice of $C$, the set $D-W$ is not connected. Hence there exists a component $W^{\prime}$ of 
bdry $W$ such that $W^{\prime}$ is not the boundary of a compact submanifold of $D$. Let $W^{\prime \prime}$ be the component of $U-W^{\prime}$ whose closure in $U$ is compact. Let $W^{\prime \prime \prime}$ be the complement of $W^{\prime} \cup W^{\prime \prime}$ in $M$. Then $W^{\prime \prime \prime}$ is a connected $n$-manifold with bdry $W^{\prime \prime \prime}=W^{\prime}$. By the way $C$ is chosen in $D$ neither $W^{\prime \prime}$ nor $W^{\prime \prime \prime}$ is contained in $E(M, h)$.

Let $x \in W^{\prime \prime} \cap N(M, h)$; by definition of $N(M, h)$, there exists a sequence of integers $\left\{\alpha_{j}\right\}_{j=1}^{\infty},\left|\alpha_{i}\right|<\left|\alpha_{i+1}\right|$ for all $i$, a sequence $\left\{y_{j}\right\}_{j=1}^{\infty} \subseteq$ $M$, a point $w \in M$ and $\delta>0$ such that

(*) $\quad \lim _{j \rightarrow+\infty} y_{j}=x, \lim _{j \rightarrow+\infty} h^{\alpha} j(x)=w \quad$ and $\quad d\left(w, \lim _{j \rightarrow+\infty} h^{\alpha}\left(y_{j}\right)\right) \geqq \delta$.

By symmetry we may assume that each $\alpha_{i}$ is positive. By replacing $\left\{\alpha_{i}\right\}$ with a subsequence, if necessary, we obtain from Lemma 2.10 a point $v \in M$ such that $\lim _{j \rightarrow+\infty} h^{\alpha}\left(W^{\prime}\right)=v$. If $U^{\prime}$ is an open $n$-cell in $M$ such that $v \in U^{\prime}$ and $J$ is chosen such that $h^{\alpha_{j}}\left(W^{\prime}\right) \subseteq U^{\prime}$ for all $j>J$, then either $h^{\alpha}\left(W^{\prime \prime}\right) \subseteq U^{\prime}$ or $h^{\alpha} j\left(W^{\prime \prime \prime}\right) \leqq U^{\prime}$ for $j>J$. Choose $U^{\prime}$ to be so small that diameter $\left(U^{\prime}\right)<\delta / 2$. By $\left({ }^{*}\right)$ we must have $h^{\alpha} j\left(W^{\prime \prime \prime}\right) \subseteq U^{\prime}$ for $j>J$ and $j$ sufficiently large. Let $k_{1}$ be one of such integers $j$.

We now proceed with $x^{\prime} \in W^{\prime \prime \prime} \cap N(M, h)$ to get, by a similar argument an open $n$-cell $U^{\prime \prime}$ in $M$ and an integer $k_{2}$ such that $h^{k_{2}}\left(W^{\prime} U\right.$ $\left.W^{\prime \prime}\right) \subseteq U^{\prime \prime}$. Then

$$
M=W^{\prime} \cup W^{\prime \prime} \cup W^{\prime \prime \prime}=h^{-k_{1}}\left(U^{\prime}\right) \cup h^{-k_{2}}\left(U^{\prime \prime}\right)
$$

and hence must be a $n$-sphere by Proposition 3.2. The contradiction establishes Proposition 3.3.

Proposition 3.4. Let $h$ be a self-homeomorphism of a closed $n$ manifold $M$ such that $N(M, h)$ is a closed nonempty set, $R(M, h)$ is zero-dimensional and $h$ is periodic at some $z \in M$; -i.e., there exists $q>0$ such that $h^{q}(z)=z$. If some component $D$ of $E(M, h)$ does not have one end, then $M-D$ consists of two points.

Proof. As in the proof of Proposition 3.3, there exists a closed connected $(n-1)$-manifold $W^{\prime}$ in $D$ such that $M-W^{\prime}$ consists of two components $W^{\prime \prime}$ and $W^{\prime \prime \prime}$ such that $W^{\prime \prime} \cap N(M, h) \neq \varnothing \neq W^{\prime \prime \prime} \cap N(M, h)$. $W^{\prime} \cup W^{\prime \prime}$ and $W^{\prime} \cup W^{\prime \prime \prime}$ are n-manifolds with boundary such that boundary $\left(W^{\prime} \cup W^{\prime \prime}\right)=W^{\prime}=$ boundary $\left(W^{\prime} \cup W^{\prime \prime \prime}\right)$. By Lemma 2.10, there exists a positive integer $m$ such that $W^{\prime} \cap h^{n}\left(W^{\prime}\right)=\varnothing$ for all integers $n$ such that $|n| \geqq m$. Let $f=h^{q m}$; note that $f(z)=z$. By Lemma 2.9, $z \notin E(M, h)$. Without loss of generality we suppose $z \in W^{\prime \prime \prime}$. There are three cases to consider.

Case 1. $f\left(W^{\prime}\right) \subseteq W^{\prime \prime}$. Hence $f\left(W^{\prime \prime}\right) \subseteq W^{\prime \prime}$, since the fixed point 
(of $f$ ) $z \in W^{\prime \prime \prime}$. It follows that the invariant sets $H=\lim _{k \rightarrow-\infty} f^{k}\left(W^{\prime}\right)$ and $K=\lim _{k \rightarrow+\infty} f^{k}\left(W^{\prime}\right)$ are connected, since each is a limit of connected sets. By using the fact that $W^{\prime} \leqq E(M, h)=E(M, f)$, one easily sees that $H=\bigcup_{x \in W^{\prime}}, \alpha(x)$ and $K=\bigcup_{x \in W^{\prime}} \omega(x)$. Then $H \cup K \subseteq R(M, f)=$ $R(M, h)$. Since $R(M, h)$ is zero-dimensional, $H$ and $K$ are fixed points of $f$. If $x \in M-\{H, K\}$, then $\lim _{k \rightarrow-\infty} f^{k}(x)=H$ and $\lim _{k \rightarrow+\infty} f^{k}(x)=K$; by [8], $E(M, h)=E(M, f)=M-\{H, K\}$.

Case 2. $W^{\prime} \subseteq f\left(W^{\prime \prime}\right)$. The argument is similar to Case 1 .

Case 3. $W^{\prime \prime} \cap f\left(W^{\prime \prime}\right)=\varnothing$. Since $W^{\prime \prime} \cap N(M, f) \neq \varnothing$, we can choose $x \in W^{\prime \prime} \cap N(M, f)$. There exists a sequence of integers $\left\{\alpha_{j}\right\}_{j=1}^{\infty}, 0<\left|\alpha_{1}\right|<$ $\left|\alpha_{2}\right|<\cdots$, a sequence $\left\{y_{j}\right\}_{j=1}^{\infty} \subseteq M, w \in M$ and $\delta>0$ such that $\lim _{j \rightarrow+\infty} y_{j}=$ $x, \lim _{j \rightarrow+\infty} f^{\alpha_{j}}(x)=w$ and $d\left(w, \lim _{j \rightarrow+\infty} f^{\alpha j}\left(y_{j}\right)\right) \geqq \delta$. Without loss of generality assume that each $\alpha_{j}$ is positive. By Lemma 2.10 there exists a subsequence $\left\{\beta_{j}=\alpha_{i_{j}}\right\}$ of $\left\{\alpha_{i}\right\}$ and $p \in M$ such that

$$
\lim _{j \rightarrow+\infty} f^{\beta j}\left(W^{\prime}\right)=p .
$$

Since the elements of $\left\{f^{n}\left(W^{\prime} \cup W^{\prime \prime}\right)\right\}_{n=+\infty}^{+\infty}$ are pairwise disjoint, it follows that $\lim _{j \rightarrow+\infty} f^{\beta_{j}}\left(W^{\prime} \cup W^{\prime \prime}\right)=p$ and hence $p=w$. But $\lim _{j \rightarrow+\infty} f^{\beta_{j}}\left(y_{j}\right)$ is also $p$ and we get a contradiction and Case 3 cannot exist.

Proof of Theorem A. By Lemma 2.9, $R(M, h) \leqq N(M, h)$. If $M$ is not the $n$-sphere, $N(M, h)$ is a continuum by Propositions 3.1 and 3.3. If $M$ is the $n$-sphere $S^{n}$ and each component of $E(M, h)$ has one end, $N(M, h)$ is a continuum by Proposition 3.1. If $n$ is even and some component of $E(M, h)$ does not have one end, then, since $h^{2}$ is orientation preserving, $h^{2}$ has a fixed point and by Proposition 3.4, $S^{n}-E\left(S^{n}, h\right)$ consists of two points which, by [7], are fixed points of $h$. If $n$ is odd and $h$ is periodic at some $z \in S^{n}$, then Proposition 3.4 and [7] again applies.

4. Structure of the ser of regular points. If $V$ is an open 2-cell in a 2-manifold $M$ and $\gamma$ is a simple closed curve in $V$, we let $I(\gamma)$ be the component of $V-\gamma$ whose closure in $V$ is compact. From the classification theory of 2-manifold, we have the following.

REMARK 4.1. An open connected 2-manifold $U$ is an open 2-cell if and only if each simple closed curve in $U$ is the boundary of a closed 2-cell in $U$.

Proof of Theorem B. Let $D$ be a component of $E(M, h)$ and let $\gamma$ be a simple closed curve in $D$. Let us first consider the case when $M$ is not the 2-sphere. It follows from Lemma 2.10 that there exists 
an open 2-cell $V$ in $M$ and an integer $k$ such that $h^{k}(\gamma) \leqq V$. If $I\left(h^{k}(\gamma)\right) \subseteq E(M, h)$, we are through by Remark 4.1. Suppose there exists $x \in I\left(h^{k}(\gamma)\right) \cap N(M, h)$; hence there exists a sequence of integers $\left\{\alpha_{j}\right\}, 0<\left|\alpha_{1}\right|<\left|\alpha_{2}\right|<\cdots$, a sequence $\left\{y_{j}\right\} \subseteq M, w \in M$ and $\delta>0$ such that

(*) $\quad \lim _{j \rightarrow+\infty} y_{j}=x, \lim _{j \rightarrow+\infty} h^{\alpha}(x)=w \quad$ and $\quad d\left(w, \lim _{j \rightarrow+\infty} h^{\alpha_{j}}\left(y_{j}\right)\right) \geqq \delta$.

Let $\gamma^{\prime}=h^{k}(\gamma)$. By Lemma 2.10, there exists a subsequence $\left\{\beta_{j}=\alpha_{i_{j}}\right\}$ of $\left\{\alpha_{i}\right\}$ such that $\lim _{j \rightarrow+\infty} h^{\beta j}\left(\gamma^{\prime}\right)=p$ for some $p \in M$. For $j$ sufficiently large, $h^{\beta j}\left(\gamma^{\prime}\right)$ bounds a closed 2-cell $B_{j}$ such that $\lim _{j \rightarrow \infty} B_{j}=p$ and $h^{\beta j}\left(I\left(\gamma^{\prime}\right)\right) \cap B_{j}=\varnothing$. Then $M=B_{j} \cup h^{\beta_{j}}\left(\gamma^{\prime} \cup I\left(\gamma^{\prime}\right)\right)$ is a 2-sphere, a contradiction.

If $M$ is a 2-sphere and $N(M, h)$ is a continuum, then by Proposition 3.4, each component of $E(M, h)$ has one end. If $D$ is a component of $E(M, h)$, then $S^{2}-D$ is connected. By [17], $D$ is an open 2-cell.

Proposition 4.2. Let $M$ and $h$ be as in Theorem A. If $U$ is a component of $E(M, h)$, the kernel of $i_{*}: \pi_{i}(U, u) \rightarrow \pi_{i}(M, u)$, the homomorphism induced on the ith homotopy groups by the inclusion map, is all of $\pi_{i}(U, u), i=1,2, \cdots$.

Proof. Let $f:\left(S^{i}, *\right) \rightarrow(U, u)$ represent an element of $\pi_{i}(U, u)$. By Lemma 2.10, there exists $p \in M$ and a subsequence $\left\{n_{j}\right\}_{i=1}^{\infty}$ of the positive integers such that $\lim _{j \rightarrow+\infty} h^{n_{j}}\left(f\left(S^{i}\right)\right)=p$. Let $V$ be an open $n$-cell in $M$ such that $p \in V$ and let $j$ be chosen such that $h^{n_{j}}\left(f\left(S^{i}\right)\right) \leqq$ $V$. Since $h^{n_{j}}\left(f\left(S^{i}\right)\right)$ is inessential in $V, f\left(S^{i}\right)$ is inessential in $h^{-n_{j}}(V) \subseteq$ $M$.

Similarly, if we use singular homology with any coefficients, we have the following.

Proposition 4.3. Let $M$ and $h$ be as in Theorem A. The homomorphism $i_{*}: H_{i}(E(M, h)) \rightarrow H_{i}(M)$ induced by inclusion is the zero homomorphism for $i=1,2, \cdots$.

Proof of Theorem C. If we consider the long exact homology sequence of the pair $(M, E(M, h))$, we get a sequence of short exact sequences by Proposition 4.3

$$
0 \longrightarrow H_{i}(M) \longrightarrow H_{i}(M, E(M, h)) \longrightarrow H_{i-1}(E(M, h)) \longrightarrow 0
$$

for $i=2,3, \cdots$. Since $M$ is connected, we get the short exact sequence

$$
0 \longrightarrow H_{1}(M) \longrightarrow H_{1}(M, E(M, h)) \longrightarrow \widetilde{H}_{0}(E(M, h)) \longrightarrow 0 \text {. }
$$

By [20; p. 296], $\bar{H}^{q}(N(M, h))$ is isomorphic to $H_{n-q}(M, E(M, h))$ and 
since we are using field coefficients, the theorem follows.

5. Open manifolds. We state a result of [12] which we need.

Proposition 5.1. Let $h$ be a self-homeomorphism of an open manifold $X$ of dimension $n \geqq 2$. If $N(X, h)$ is compact and zerodimensional, then $X$ is homeomorphic to n-dimensional Euclidean space and $N(X, h)$ consists of a fixed point.

Proof of Theorem D. Let $M^{*}=M \cup\{\infty\}$ be the one point compactification of $M$. Note that, in general $M^{*}$ is not a manifold and so Theorem A does not apply. Let $h^{*}$ be the extension of $h$ to $M^{*}$. Let $W$ be a compact neighborhood of $\infty$ such that $W \cap N(M, h)=\varnothing$. Using the compactness of bdry $W$, one easily sees that the number of components of $E(M, h)$ which intersects bdry $W$ is at most finite. One also observes that $N(M, h)$ meets the closure of every component of $E(M, h)$. It follows readily that there exists a component $G$ of $E(M, h)$ such that $\infty \in \mathrm{Cl}(G)$ and $\mathrm{Cl}(G) \cap N(M, h) \neq \varnothing$, where $\mathrm{Cl}(G)$ is the closure of $G$ in $M^{*}$. Since $\{\infty\}$ and $N(M, h)$ are invariant under $h^{*}$ and $G$ is connected, $h^{i}(G) \cap$ bdry $W \neq \varnothing$ for all integers $i$. Hence there exists a positive integer $k$ such that $h^{k}(G)=G$. Let $X$ be the decomposition space obtained from $\mathrm{Cl}(G)$ by shrinking the components of $N(M, h) \cap \mathrm{Cl}(G)$ to points and if $\phi: \mathrm{Cl}(G) \rightarrow X$ is the natural projection, then define $f: X \rightarrow X$ so that $f \circ \phi=\phi \circ\left(h^{*}\right)^{k}$. Since $\phi$ is uniformly continuous and $\phi \mid G$ is open, $E(X, f) \supseteqq \phi(G)$. By Lemma 2.10 , applied to the space $\mathrm{Cl}(G)$, we easily see that $N(X, f) \neq \varnothing$. By Theorem 2.11, $N(X, f)$ consists of the union of one or two fixed points of $f$ and

$$
\lim _{n \rightarrow+\infty} f^{n}(x)=p, \lim _{n \rightarrow-\infty} f^{n}(x)=q \text { for all } x \in E(X, f),
$$

where $N(X, f)=\{p, q\}$ and $p$ may be equal to $q$. Without loss of generality we may assume that $\phi(\infty)=p$. We show that under the present hypothesis $q \neq p$. Since $\infty \notin N(M, h) \cap \mathrm{Cl}(G)$, it follows that if $y \in \phi(N(M, h) \cap \mathrm{Cl}(G))$, then $\phi(\infty)$ is not in the orbit closure of $y$. Hence we must have $q=\phi(N(M, h) \cap \mathrm{Cl}(G))$ by (a). This shows $q \neq p$ and that $N(M, h) \cap \mathrm{Cl}(G)$ is connected. We have two cases to consider.

Case (i). $\quad N(M, h) \cap \mathrm{Cl}(G)=\{q\}$, a singleton. Since a point cannot separate $n$-manifold, $n \geqq 2, M=G \cup\{q\}$ and the theorem follows from Proposition 5.1.

Case (ii). $\quad N(M, h) \cap \mathrm{Cl}(G)$ is a nondegenerate continuum $A$. By Proposition 3.1, $G$ does not have one end. There then exists a compact 
set $C \cong G$ such that for each compact set $K, C \subseteq K \subseteq G, G-K$ is not connected. By (a) we have $\lim _{i \rightarrow+\infty} h^{k i}(C)=\infty$ and

$$
\lim _{i \rightarrow-\infty} \sup h^{k i}(C)=A \text {. }
$$

Applying Lemma 2.10 to $M^{*}$ and $\left(h^{*}\right)^{k}$, there exists a point $w \in A$ and a subsequence $\left\{n_{j}\right\}_{j=-\infty}^{+\infty}$ of the integers such that $\lim _{j \rightarrow+\infty} h^{k n_{j}}(C)=\infty$ and $\lim _{j \rightarrow-\infty} h^{k n_{j}}(C)=w$. The second equation implies that $h^{m}(C)$ lies in an open $n$-cell $U$ in $M$ for an integer $m$. As in the proof of Proposition 3.3 we obtain some closed connected $(n-1)$-manifold $W^{\prime} \subseteq G$ such that the two complementary components $W^{\prime \prime}$ and $W^{\prime \prime \prime}$ of $M^{*}-W^{\prime}$ have $W^{\prime}$ as their common boundary and neither $W^{\prime \prime}$ nor $W^{\prime \prime \prime}$ is contained in $E\left(M^{*}, h\right)$. Note that $R\left(M^{*},\left(h^{*}\right)^{k}\right)=R\left(M^{*}, h^{*}\right)$ is zero-dimensional and $\left(h^{*}\right)^{k}$ has a fixed point $\infty$. As in the proof of Proposition 3.4 we have three cases. Case 1. $\left(h^{*}\right)^{k}\left(W^{\prime}\right) \subseteq W^{\prime \prime}$. Case 2. $W^{\prime} \subseteq\left(h^{*}\right)^{k}\left(W^{\prime \prime}\right)$. Case 3. $W^{\prime \prime} \cap\left(h^{*}\right)^{k}\left(W^{\prime \prime}\right)=\varnothing$. The argument for Proposition 3.4 for these three cases also applies here, though $M^{*}$ now fails to be locally Euclidean at the point $\infty$. Hence Case 3 can be shown to be impossible under the given hypothesis and either one of Cases 1 and 2 implies that $N(M, h)$ is a singleton which also leads to a contradiction. Hence Case (ii) cannot occur. Theorem D follows.

6. Examples. We give some examples here to justify our results. Examples are to be given for dimensions 2 and 3, but higher dimensional ones can be constructed from these models. First Theorems $\mathrm{A}$ and $\mathrm{D}$ would fail if the restriction on nonwandering points is omitted.

EXAMPLE 6.1. Let points in the plane $\boldsymbol{R}^{2}$ be given representation of complex numbers:

$p=(n+r) e^{i \theta} ; n:$ nonnegative integers, $0 \leqq r<1,0 \leqq \theta<2 \pi$. Define a homeomorphism $g: \boldsymbol{R}^{2} \rightarrow \boldsymbol{R}^{2}$ by

$$
g(p)=\left(n+r^{2}\right) e^{i \theta} \text { for } p=(n+r) e^{i \theta} .
$$

Then $N\left(\boldsymbol{R}^{2}, g\right)$ consists of the circles $C_{n} ; n=0,1,2, \cdots$, where $C_{n}=\{p \in$ $\left.R^{2}|| p \mid=n\right\}$. Hence $N\left(\boldsymbol{R}^{2}, g\right)$ has infinitely many components. By adding the point at infinity to get $S^{2}$ and by extending $g$ naturally to $S^{2}$, we have that $N\left(S^{2}, g\right)$ has infinitely many components.

The next three examples show the generality of the class of homeomorphisms which satisfy Theorem A or Theorem D.

EXAMPLe 6.2. Let $I$ be the closed unit interval $[0,1]$ and let $f$ : $I \rightarrow I$ be an order preserving self-homeomorphism whose fixed point set is $\{0,1\}$. Note that $f$ is regular except on $\{0,1\}$. Let $g$ be the 
product homeomorphism on $I \times I$. Then the set $N(I \times I, g)$ equals the boundary of $I \times I$. By identifying points $(x, 0)$ with $(x, 1)$ and $(0, y)$ with $(1, y)$ for all $|x| \leqq 1,|y| \leqq 1$ the set $I \times I$ becomes a torus $S^{1} \times S^{1}$ and $g$ induces a homeomorphism $h$ on $S^{1} \times S^{1}$. It is readily verified that $N\left(S^{1} \times S^{1}, g\right)$ is the union of a vertical circle with a horizontal circle if the torus $S^{1} \times S^{1}$ is placed stably on a table. Note that the intersection of the two circles is the only nonwandering point of the torus. This process can be modified to get a homeomorphism of any closed 2-manifold which fails to be regular on a one-dimensional polyhedron and with a finite nonwandering set.

The set of regular points in Example 6.2 is connected. The next example is one such that this set has infinitely many components.

ExAmple 6.3. We take the example of Massey [16, p. 167]. Consider the system of differential equations in the $(x, y)$ plane:

$$
\left\{\begin{array}{l}
\frac{d x}{d t}=\cos ^{2} x \\
\frac{d y}{d t}=\sin x .
\end{array}\right.
$$

The integral curves are then the curves $y=\sec x+c$. A continuous flow on the plane is then generated from the autonomous system of differential equations. The acting group is then the real numbers. Restricting to the integers, we then have a discrete flow. Call the generating homeomorphism $h$. It is readily verified that $N\left(\boldsymbol{R}^{2}, h\right)$ is the union of all vertical lines whose $x$-coordinates are integers. By considering the 2 -sphere as the one-point compactification of $\boldsymbol{R}^{2}$, we see that the extended homeomorphism $h$ on $S^{2}$ is such that $N\left(S^{2}, h\right)=$ $\{\infty\} \cup N\left(\boldsymbol{R}^{2}, h\right)$ and $\infty$ is the only nonwandering point in $S^{2}$ (see figure on p. 168 of [16]).

The number of nonwandering points in the previous examples are finite. The next example is such that the nonwandering points are infinite and zero-dimensional.

EXAMPLE 6.4. Let $h$ be a self-homeomorphism of the unit circle $C$ which has neither periodic points nor dense orbits. It is then known that the discrete flow on $C$ generated by $h$ has a unique minimal set $K$ which is homeomorphic to the Cantor set (cf. for example [15]). $I$ is a complementary interval of $K$, then $I \cap\left(h^{n}(I)\right)=\varnothing$ for all integers $n \neq 0$. It follows from argument of finite measure that $\lim _{|n| \rightarrow \infty}$ diameter $\left[h^{n}(I)\right]=0$. Hence $I \subseteq E(C, h)$. Since $K$ is a minimal set, by Proposition 2.2 (1) we have either $K \subseteq E(C, h)$ or $K \cong N(C, h)$. The first alternative is impossible, since it is well-known that a homeomorphism 
regular everywhere in $C$ is topologically conjugate to either a rotation or to a reflection with respect to the $x$-axis. The assumption for $h$ shows that it is impossible.

We let $A(z)$ denote the segment which joins the origin to a point $z \in C$. Extend $h$ to the entire unit disc by requiring that $A(H(z))=$ $h(A(z)), z \in C$. It is clear that $N(D, h)=\bigcup\{A(z) \mid z \in K\}-\{0\}$, where $D$ is the unit disc. Every point in $N(D, h)$ is nonwandering.

We next modify the homeomorphism $h$ as follows. Let $g: D \rightarrow D$ be the restriction of the homeomorphism given in Example 6.1. We let $f=g \circ h=h \circ g$. It is readily verified that

$$
N(D, f)=N(D, h) \cup\{0\}=\bigcup\{A(z) \mid z \in K\} .
$$

The only nonwandering points of $f$ are the origin and points of $K$.

Finally the homeomorphism $f$ can be extended to the 2-sphere by combining two discs. The resulting homeomorphism has a connected one-dimensional $N\left(S^{2}, f\right)$ and its set of nonwandering points is uncountable and zero-dimensional.

Example 6.5. Define a homeomorphism $h^{\prime}$ of Euclidean 3-space $\boldsymbol{R}^{3}$ by

$$
h^{\prime}(x, y, z)= \begin{cases}(x / 2, y / 2, z / 2) & x \geqq 0 \\ (2 x, y / 2, z / 2) & x \leqq 0\end{cases}
$$

and extend $h^{\prime}$ to a homeomorphism $h$ of $S^{3}=\boldsymbol{R}^{3} \cup\{\infty\}$ by defining $h(\infty)=\infty$. Note that $N\left(S^{3}, h\right)=\{(x, y, z) \mid x=0$ or $y=z=0\} \cup\{\infty\}$ and $R\left(S^{3}, h\right)=\{(0,0,0), \infty\}$. Let $D_{i}=\left\{(x, y, z) \mid x^{2}+y^{2}+z^{2}=2^{2 i}\right.$ and $x \geqq 0\}$ where $i$ is an integer; note that $h^{\prime}\left(D_{i}\right)=D_{i-1}$ for all $i$. Let $\phi:[0,1] \rightarrow R^{3}$ be an embedding such that $\phi(0)=(0,0,3 / 2)$, the $x$ and $z$ coordinates of $\phi(t)$ for $0<t \leqq 1$ are positive, $\phi([0,1])$ is locally tame except at $\phi(1)$, and $\phi([0,1]) \cap D_{i}=\varnothing$ for all $i$. Let $\xi:[0,1] \rightarrow \boldsymbol{R}^{3}$ be an embedding such that $\xi(0)=(0,0,-3 / 2)$, the $x$ and $z$ coordinates of $\xi(t)$ for $0<t \leqq 1$ are positive and negative respectively, $\xi([0,1])$ is locally tame except at $\xi(1)$ and $\xi([0,1]) \cap D_{i}=\varnothing$ for all $i$. By [19], $h^{i} \xi([0,1])$ and $h^{i} \phi([0,1])$ are cellular subsets of $S^{3}$ for each $i$. Let $X$ be the decomposition space obtained by shrinking the components of $\left\{D_{i}, h^{i} \xi([0,1]), h^{i} \phi([0,1]) ;-\infty<i<+\infty\right\}$ to points and let $p: S^{3} \rightarrow X$ be the natural projection. By [1], $X$ is homeomorphic to $S^{3}$. Define $f: X \rightarrow X$ such that $f \circ p=p \circ h$; note that $f$ is a homeomorphism of $X$ onto itself such that $N(X, f)=p N\left(S^{3}, h\right)$ and $R(X, f)=p R\left(S^{3}, h\right)$. If $U=\left\{(x, y, z) \mid 1 \leqq x^{2}+y^{2}+z^{2} \leqq 2^{2}\right.$ and $\left.x \geqq 0\right\}$, then the components of $X-N(X, f)$ are int $f^{i}(p U),-\infty<i<+\infty$, and $p(\{(x, y, z) \mid x<0\}-$ $\{(x, y, z) \mid y=z=0\})$. By [19], int $f^{i}(p U)$ is not an open 3-cell and if we choose $\phi$ and $\xi$ such that $\pi_{1}(U-(\phi([0,1]) \cup \xi([0,1])))$ is infinitely 
generated [5], $\pi_{1}$ (int $f^{i}(p U)$ ) would also be infinitely generated. Hence it appears that there is not hope of characterizing the components of $E(M, h)$ in higher dimensions as is done in Theorem $\mathrm{B}$ for dimension 2.

\section{REFERENCES}

1. R. H. Bing, Upper semicontinuous decompositions of $E^{3}$, Ann. of Math., 65 (1957), 363-374.

2. M. Brown, A proof of the generalized Schoenflies theorem, Bull. Amer. Math. Soc., 66 (1960), 74-76.

3. J. Dugundji, Topology, Allyn and Bacon, Boston, 1966.

4. P. F. Duvall, Jr. and L. S. Husch, On the homotopy type of irregular sets, Proc. Amer. Math. Soc., 38 (1973), 419-422.

5. R. H. Fox and E. Artin, Some wild cells and spheres in three dimensional space, Ann. of Math., 49 (1948), 979-990.

6. W. H. Gottschalk and G. A. Hedlund, Topological Dynamics, Amer. Math. Soc. Colloq. Publ., Vol. 36 Amer. Math. Soc., Providence, R. I., 1955.

7. T. Homma and S. Kinoshita, On homeomorphisms which are regular except for a finite number of points, Osaka Math. J., 7 (1955), 29-38.

8. - On the regularity of homeomorphisms of $E^{n}$, J. Math. Soc., Japan, 5 (1953), $365-371$.

9. S. K. Kaul, On almost regular homeomorphisms, Canad. J. Math., 20 (1968), 1-6.

10. B. V. Kerékjártó, Topologische charakterisierung der linearen Abbildungen, Acta Sci. Math., Szeged, 6 (1934), 235-262.

11. S. Kinoshita, On quasi-translation in 3-space, Fund. Math., 56 (1964), 69-79.

12. P.-F. Lam, On a theorem of B. von Kerékjártó, Bull. Amer. Math. Soc., 77 (1971), 130-234.

13. - Equicontinuity and indivisibility in transformation groups, Trans. Amer. Math. Soc., (to appear).

14. - Almost equicontinuous transformation groups, (to appear).

15. N. G. Markley, Transitive homeomorphisms of the circle, Math. Systems Th., 2 (1968), 247-249.

16. W. S. Massey, Algebraic Topology, Harcourt, Brace and World, New York, 1967.

17. R. L. Moore, Concerning upper semicontinuous collections of continua, Trans. Amer. Math. Soc., 27 (1925), 416-428.

18. F. A. Roberson, A theorem on near equicontinuity of transformation groups, Proc. Amer. Math. Soc., 27 (1971), 189-191.

19. C. D. Sikkema, A duality between certain spheres and arcs in $S^{3}$, Trans. Amer. Math. Soc., 122 (1966), 399-415.

20. E. H. Spanier, Algebraic Topology, McGraw-Hill Book Co., New York, 1966.

Received July 14, 1972 and in revised form July 11, 1973. Research of the first author was supported in part by NSF Grant GP-33872.

UNIVERSITY OF TENNESSEE

UNIVERSITY OF MISSOURI

AND

Institute for Advanced Study 


\section{PACIFIC JOURNAL OF MATHEMATICS}

\section{EDITORS}

RICHARD ARENS (Managing Editor) University of California

Los Angeles, California 90024

R. A. BeaUmont

University of Washington Seattle, Washington 98105
J. DUGUNDJI*

Department of Mathematics University of Southern California Los Angeles, California 90007

D. Gilbarg and J. Milgram Stanford University

Stanford, California 94305

\section{ASSOCIATE EDITORS}

E. F. BECKENBACH

B. H. NeUmanN

F. WOLF

K. YoSHIDA

\section{SUPPORTING INSTITUTIONS}

\section{UNIVERSITY OF BRITISH COLUMBIA CALIFORNIA INSTITUTE OF TECHNOLOGY UNIVERSITY OF CA.LIFORNIA MONTANA STATE UNIVERSITY UNIVERSITY OF NEVADA NEW MEXICO STATE UNIVERSITY OREGON STATE UNIVERSITY UNIVERSITY OF OREGON OSAKA UNIVERSITY}

UNIVERSITY OF SOUTHERN CALIFORNIA STANFORD UNIVERSITY UNIVERSITY OF TOKYO UNIVERSITY OF UTAH WASHINGTON STATE UNIVERSITY UNIVERSITY OF WASHINGTON AMERICAN MATHEMATICAL SOCIETY NAVAL WEAPONS CENTER

* C. R. DePrima California Institute of Technology, Pasadena, CA 91109, will replace J. Dugundji until August 1974. 


\section{Pacific Journal of Mathematics}

\section{Vol. 50, No. $1 \quad$ September, 1974}

Gail Atneosen, Sierpinski curves in finite 2-complexes.............. 1

Bruce Alan Barnes, Representations of $B^{*}$-algebras on Banach spaces .... 7

George Benke, On the hypergroup structure of central $\Lambda(p)$ sets ....... 19

Carlos R. Borges, Absolute extensor spaces: a correction and an

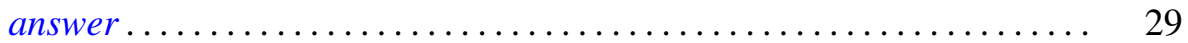

Tim G. Brook, Local limits and tripleability .................. 31

Philip Throop Church and James Timourian, Real analytic open maps .... 37

Timothy V. Fossum, The center of a simple algebra ............... 43

Richard Freiman, Homeomorphisms of long circles without periodic

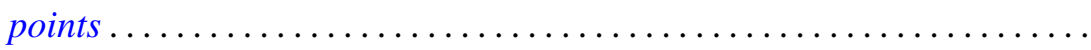

B. E. Fullbright, Intersectional properties of certain families of compact

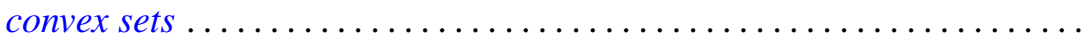

Harvey Charles Greenwald, Lipschitz spaces on the surface of the unit

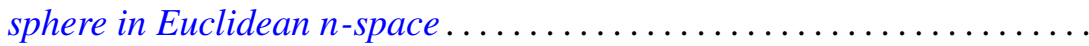

Herbert Paul Halpern, Open projections and Borel structures for

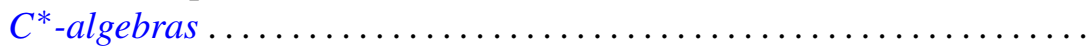

Frederic Timothy Howard, The numer of multinomial coefficients divisible

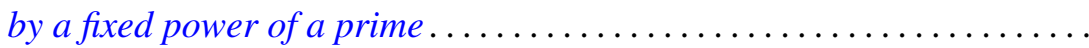

Lawrence Stanislaus Husch, Jr. and Ping-Fun Lam, Homeomorphisms of manifolds with zero-dimensional sets of nonwandering points........ 109

Joseph Edmund Kist, Two characterizations of commutative Baer rings ...

Lynn McLinden, An extension of Fenchel's duality theorem to saddle functions and dual minimax problems ................

Leo Sario and Cecilia Wang, Counterexamples in the biharmonic classification of Riemannian 2-manifolds...

Saharon Shelah, The Hanf number of omitting complete types ...

Richard Staum, The algebra of bounded continuous functions into a

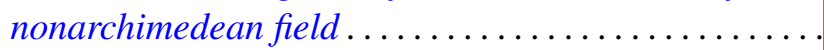

James DeWitt Stein, Some aspects of automatic continuity ..

Tommy Kay Teague, On the Engel margin

John Griggs Thompson, Nonsolvable finite groups all of whose local subgroups are solvable, $V \ldots \ldots \ldots \ldots \ldots \ldots \ldots \ldots$

Kung-Wei Yang, Isomorphisms of group extensions 